\title{
Commentary: Complex tumors of the glomus jugulare: criteria, treatment, and outcome. J Neurosurg 97:1356-1366, 2002
}

\author{
Ossama Al-Mefty, M. D. \\ Department of Neurosurgery, University of Arkansas for Medical Sciences, Little Rock, Arkansas
}

With rare exceptions, lesions of the glomus jugulare are benign, slow-growing paragangliomas. Advances in neuroimaging, skull base techniques, embolization, and anesthetic agents, along with emphasis on postoperative care have markedly improved the surgeons' ability to remove these tumors and have improved outcome in an overwhelming number of patients who present with tumors of the glomus jugulare. Studies that include long-term follow up show the effectiveness of total removal in achieving a cure.

There remains, however, a rare subgroup of glomus jugulare tumors that continue to present a formidable challenge to treatment. We have used the term "complex glomus jugulare tumors" to classify this group. These tumors are frequently considered to be extremely high risk or inoperable. One or more of the following criteria should be met to qualify a case for this designation: giant size, multiple paragangliomas (bilateral or ipsilateral), malignancy, catecholamine secretion, or association with other lesions such as dural arteriovenous malformations or an adrenal tumor. Previous treatment that resulted in adverse outcomes such as sacrifice of the carotid artery, necessity for radiation therapy, postoperative deficit, or adverse effect from embolization makes surgical intervention much more risky.

We undertook the management of these tumors and report on the challenges involved in surgical removal, with commentary on various techniques and their outcomes. We emphasize the perioperative management of these lesions in special situations such as hypersecretion, multiple paragangliomas, and vascular involvement, and we discuss the rare malignant behavior of some of these tumors. 


\title{
Complex tumors of the glomus jugulare: criteria, treatment, and outcome
}

\author{
Ossama Al-Mefty, M.D., AND Aramis TeiXeira, M.D. \\ Department of Neurosurgery, University of Arkansas for Medical Sciences, Little Rock, Arkansas
}

\begin{abstract}
Object. Tumors of the glomus jugulare are benign, slow-growing paragangliomas. Their natural history, surgical treatment, and outcome have been well addressed in the recent literature; however, there remains a subgroup of complex tumors-multiple, giant, malignant, neuropeptide-secreting lesions, and those treated previously by an intervention with an adverse outcome - that is high risk, presents surgical challenges, and is associated with treatment controversy. In this article the authors report on a series of patients with complex glomus jugulare tumors and focus on treatment decisions, avoidance of complications, surgical refinements, and patient outcomes.

Methods. In this retrospective study, the patient population was composed of 11 male and 32 female patients (mean age 47 years) with complex tumors of the glomus jugulare who were treated by the senior author within the past 20 years. These include 38 patients with giant tumors, 11 with multiple paragangliomas (seven bilateral and four ipsilateral), two with tumors that hypersecreted catecholamine, and one with a malignant tumor. Six patients had associated lesions: one dural arteriovenous malformation, one carotid artery (CA) aneurysm, two adrenal tumors, and two other cranial tumors.

All but one patient presented with neurological deficits. Cranial nerve deficits, particularly those associated with the lower cranial nerves, were the prominent feature. Twenty-eight patients underwent resection in an attempt at total removal, and gross-total resection was achieved in 24 patients. Particularly challenging were cases in which the patient had undergone prior embolization or CA occlusion, after which new feeding vessels from the internal CA and vertebrobasilar artery circulation developed.

The surgical technique was tailored to each patient and each tumor. It was modified to preserve facial nerve function, particularly in patients with bilateral tumors. Intrabulbar dissection was performed to increase the likelihood that the lower cranial nerves would be preserved. Each tumor was isolated to improve its resectability and prevent blood loss. No operative mortality occurred. In one patient hemiplegia developed postoperatively due to CA thrombosis, but the patient recovered after an endovascular injection of urokinase. In four patients a cerebrospinal fluid leak was treated through spinal drainage, and in five patients infection developed in the external ear canal. Two of these infections progressed to osteomyelitis of the temporal bone. There were two recurrences, one in a patient with a malignant tumor who eventually died of the disease.

Conclusions. Despite the challenges encountered in treating complex glomus jugulare tumors, resection is indicated and successful. Multiple tumors mandate a treatment plan that addresses the risk of bilateral cranial nerve deficits. The intrabulbar dissection technique can be used with any tumor, as long as the tumor itself has not penetrated the wall of the jugular bulb or infiltrated the cranial nerves. Tumors that hypersecrete catecholamine require perioperative management and malignant tumors carry a poor prognosis.
\end{abstract}

\section{KEY WORDS - brain neoplasm - skull base tumor - carotid body • catecholamine • paraganglioma $\bullet$ glomus jugulare}

$\mathrm{W}$ ITH rare exceptions, tumors of the glomus jugulare are benign, slow-growing paragangliomas. Since the first documentation of removal of this type of lesion by Rosenwasser in $1945,{ }^{51}$ these tumors continue to present a treatment challenge accompanied by marked controversy. As recently as 1978, Simko and colleagues ${ }^{57}$ stated that complete surgical excision of these lesions is seldom possible because of their vascularity, invasive character, and critical location, and often because of the advanced stage of the tumor at presentation. Nevertheless, advances in neuroimaging, skull base techniques, embolization, anesthesia, and emphasis on postoperative care have markedly im-

Abbreviations used in this paper: $\mathrm{CA}=$ carotid artery; $\mathrm{CT}=$ computerized tomography; ICA = internal $\mathrm{CA} ; \mathrm{JV}=$ jugular vein; $\mathrm{MR}=$ magnetic resonance. proved the surgeon's ability to remove these tumors and have improved outcome in an overwhelming number of patients with tumors of the glomus jugulare. ${ }^{20,23,25,65}$ Studies with long-term follow up show the effectiveness of total removal in achieving a cure. $22,40,49$

There remains, however, a subgroup of glomus jugulare tumors that is rarely encountered and seldom reported, although these tumors continue to present a formidable challenge to treatment. We classified this group under the term "complex glomus jugulare tumors." These tumors are frequently considered extremely high risk or inoperable. ${ }^{11,20,31,58}$ To qualify for this category, one or more of the following criteria must be met: giant size, multiple paragangliomas (bilateral or ipsilateral), malignancy, catecholamine secretion, association with other lesions such as a dural arteriovenous malformation or an adrenal tumor, or previous treat- 
TABLE 1

Criteria for diagnosis of complex glomus jugulare tumors in 43 patients*

\begin{tabular}{lcc}
\hline \hline Complexity Factor & $\begin{array}{c}\text { Total No. } \\
\text { of Cases } \\
\text { (43 patients) }\end{array}$ & $\begin{array}{c}\text { No. of Sur- } \\
\text { gically Treated } \\
\text { Patients (28 cases) }\end{array}$ \\
\hline giant tumor & 38 & 23 \\
multiple paragangliomas & 11 & 9 \\
bilat tumors & 7 & 5 \\
ipsilat tumors & 4 & 4 \\
malignant tumor & 1 & 1 \\
hypersecreting tumor & 2 & 2 \\
associated lesions & 6 & 4 \\
dural AVM & 1 & 1 \\
CA aneurysm & 1 & 1 \\
adrenal tumor & 2 & 2 \\
other cranial tumor & 2 & 0 \\
prior treatment w/ adverse outcome & 19 & 18 \\
cerebrovascular occlusion & 4 & 4 \\
radiation therapy & 5 & 5 \\
surgically induced deficit & 8 & 7 \\
embolization-induced adverse effect & 2 & 2 \\
\hline
\end{tabular}

* AVM = arteriovenous malformation.

ment with adverse outcome that makes surgical intervention a much higher risk, such as sacrifice of the CA, radiation therapy, and postoperative deficits or adverse effects from embolization.

\section{Clinical Material and Methods}

We reviewed a database of patients with pathological conditions of the skull base treated by the senior author to identify those patients with complex glomus jugulare tumors. Forty-three patients fulfilled one or more of the criteria for these tumors: 38 patients harbored giant tumors, 11 had multiple paragangliomas (bilateral in seven cases and ipsilateral in four cases), two had tumors with hypersecretion of catecholamine, one had a malignant paraganglioma, six had associated lesions, and 19 had undergone previous treatments with adverse outcomes (surgical, radiation, or embolization) that increased the surgical risk because neurovascular structures had been compromised (Table 1). Tumors of the glomus jugulare that did not meet the criteria of complex tumors were excluded because their treatment and outcomes have been well described in the literature. The patients in our series were treated between July 1982 and June 2001 at four institutions: King Faisal Specialist Hospital in Riyadh, Saudi Arabia; the University of Mississippi Medical Center; Loyola University Medical Center in Chicago; and the University of Arkansas for Medical Sciences. A few of the patients have been included in previous case reports or descriptions of surgical technique. $2,5,8$

The medical record (hospital, clinic, department, and follow-up notes) of each patient was reviewed. Twenty-eight patients underwent surgical treatment. The remaining patients either declined surgery and received radiation therapy at other institutions or were followed up conservatively. Patients were evaluated through detailed neurological, neuroophthalmological, and neurootological examinations. High-resolution CT scans of the temporal bone with a bone algorithm were obtained. Multiplanar MR images were obtained both with and without contrast enhancement (nine
TABLE 2

Clinical findings in 43 patients with complex glomus jugulare tumors

\begin{tabular}{|c|c|}
\hline Finding & No. of Cases \\
\hline hoarseness & 19 \\
\hline hearing deficits & 16 \\
\hline dizziness & 9 \\
\hline difficulty w/ swallowing & 9 \\
\hline pulsatile tinnitus & 9 \\
\hline hypertension & 9 \\
\hline headache & 8 \\
\hline sternocleidomastoid atrophy or weakness & 6 \\
\hline tongue atrophy or fasciculation & 4 \\
\hline neck pain & 5 \\
\hline ear pain & 2 \\
\hline ear mass & 2 \\
\hline ear bleeding & 2 \\
\hline diplopia & 2 \\
\hline
\end{tabular}

patients were treated before MR imaging was available). In each patient the serum catecholamine level was measured routinely, as were urine levels of vanillylmandelic acid and metanephrines. Computerized tomography scans of the abdomen were obtained to look for associated adrenal lesions. The vascular anatomy and the blood supply were delineated through cerebral angiography, and cerebral collateral blood flow was assessed through cross-compression studies. Only two patients underwent a balloon occlusion test. In $20 \mathrm{pa}-$ tients, superselective tumor embolization was performed using polyvinyl alcohol, which was injected through the branches of the external CA prior to surgery. Follow-up CT scans were obtained immediately after surgery. Postoperatively, MR images were acquired early after surgery; follow-up images were obtained at 6-month intervals for the 1 st year and later on an annual basis. The follow-up period was defined as the period extending from surgery to the most recent clinical visit or patient contact (mean 38 months, range $2-108$ months).

\section{Results}

Among these 43 patients were 32 female and 11 male patients ranging in age from 11 to 86 years (mean 47 years). All but one patient presented with neurological deficits. Clinical findings are presented in Table 2. Cranial nerve deficits, particularly those associated with the lower cranial nerves, were the prominent feature (Table 3).

All tumors were clearly identified on the imaging studies. The CT, MR imaging, and angiographic findings were diagnostic for paragangliomas. Multiple tumors were also depicted on MR imaging studies, except for one small sphenopalatine paraganglioma that was seen only on an angiogram. Angiographic studies were critical for assessing the appropriateness of preoperative embolization after the tumor's blood supply had been demonstrated. The most critical aspect of the angiographic evaluation in patients who had undergone previous embolization or CA occlusion was the identification of new feeding vessels from the ICA and vertebrobasilar artery circulation.

\section{Surgical Considerations}

Twenty-eight patients underwent resection in an attempt 
TABLE 3

Cranial nerve deficits at presentation

\begin{tabular}{lc}
\hline \hline Cranial Nerve Deficit & No. of Cases \\
\hline II & 2 \\
III & 2 \\
IV & 1 \\
V & 10 \\
VI & 7 \\
VII & 18 \\
VIII & 19 \\
IX & 23 \\
X & 31 \\
XI & 17 \\
XII & 23 \\
Horner syndrome & 4 \\
\hline
\end{tabular}

at total tumor removal. Gross-total removal was determined intraoperatively and confirmed by postoperative MR images in 24 patients. Four patients harbored small residual tumors. The surgical approach was tailored according to the patient's anatomy, condition, and deficits (Table 4). Transposition of the facial nerve was avoided in all patients with bilateral lesions and was necessary in only five cases. Three technical maneuvers deserve particular emphasis and are described in the following subsections.

Intrabulbar Dissection. Intrabulbar dissection is a maneuver that facilitates preservation of the lower cranial nerves. This maneuver can be used for any tumor, as long as the tumor itself has not penetrated the wall of the jugular bulb or actually infiltrated the cranial nerves.

A skull base approach that skeletonized the sigmoid sinus, jugular bulb, and facial nerve was used. After the upper neck had been dissected and the ninth through 12th nerves
TABLE 4

Surgical approaches in 28 patients

\begin{tabular}{lc}
\hline \multicolumn{1}{c}{ Approach } & No. of Cases \\
\hline infratemporal & 3 \\
combined infratentorial \& posterior fossa & 22 \\
cranioorbitozygomatic & 2 \\
combined approaches w/ total petrosectomy & 1 \\
intrabulbar dissection & 11 \\
\hline
\end{tabular}

had been exposed along the JV, the sigmoid sinus was ligated distally and the JV was ligated proximally. The outer wall of the lower sigmoid sinus was incised along the jugular bulb into the JV. The tumor was then removed from inside the jugular bulb and the sigmoid sinus, and the tail end of the JV was separated from the lower cranial nerves (Fig. 1). The innermost venous wall separating the tumor from the nerves was left in situ to minimize dissection, traumatization, manipulation, or devascularization of the lower cranial nerves. We used this procedure in the last 11 cases. Using this technique, we were able to preserve the immediate postoperative function of the lower cranial nerves in four patients in whom the tumor did not transgress the venous wall at the jugular foramen.

Isolation of Giant Tumors. Each tumor was totally isolated for safe surgical removal. This is best done in one stage through the combined posterior fossa and infratemporal approach. ${ }^{2}$ This approach allows the tumor to be devascularized from the intrapetrous CA. It also is used to separate the tumor from the posterior fossa and dissect the lower portion from the nerves with minimal blood loss while preserving the vessels (Fig. 2).

Arterial and Venous Handling. Resection of tumors of the

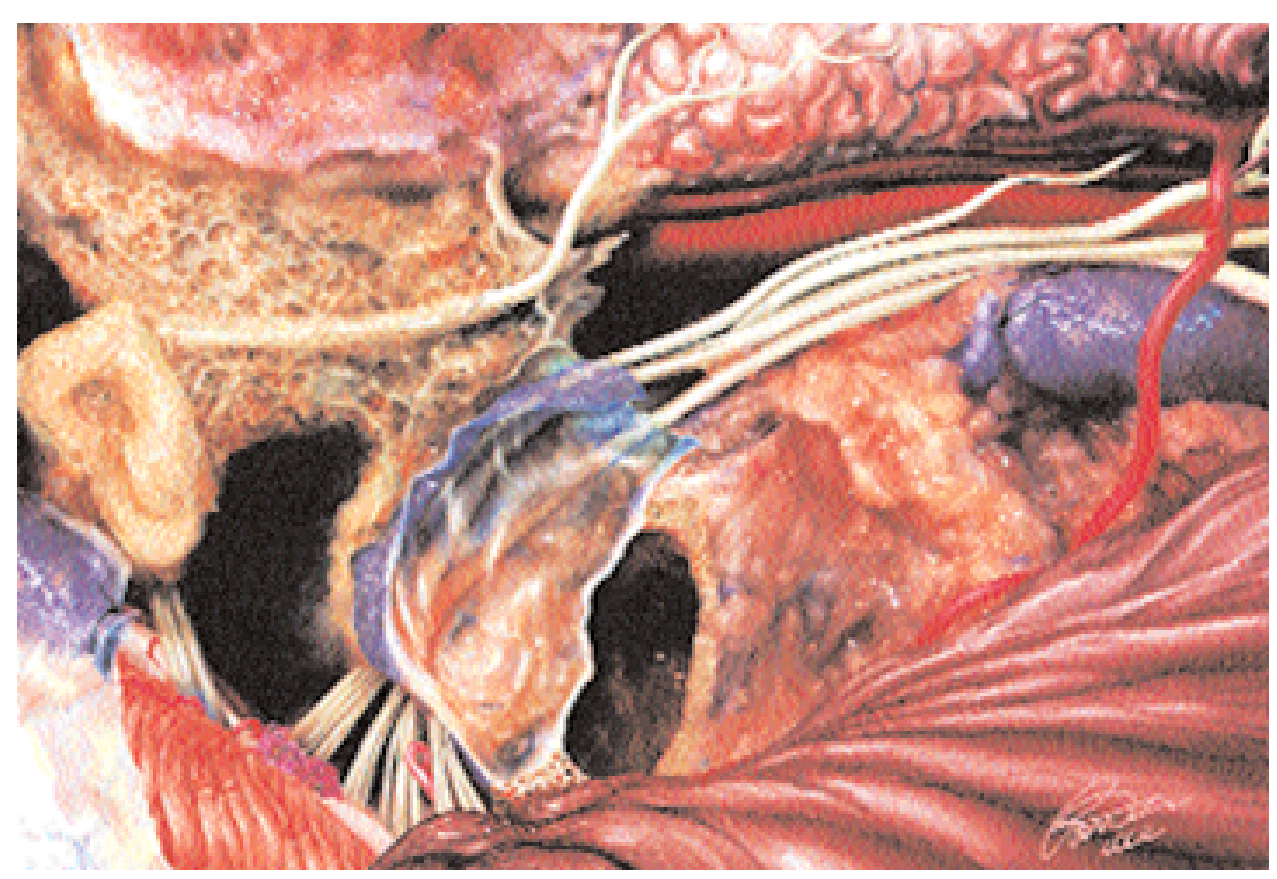

FIG. 1. Artist's illustration demonstrating intrabulbar dissection performed by preserving the anterior and medial walls of the jugular bulb against the lower cranial nerves. This dissection minimizes manipulation and improves the chance of preserving these nerves. 


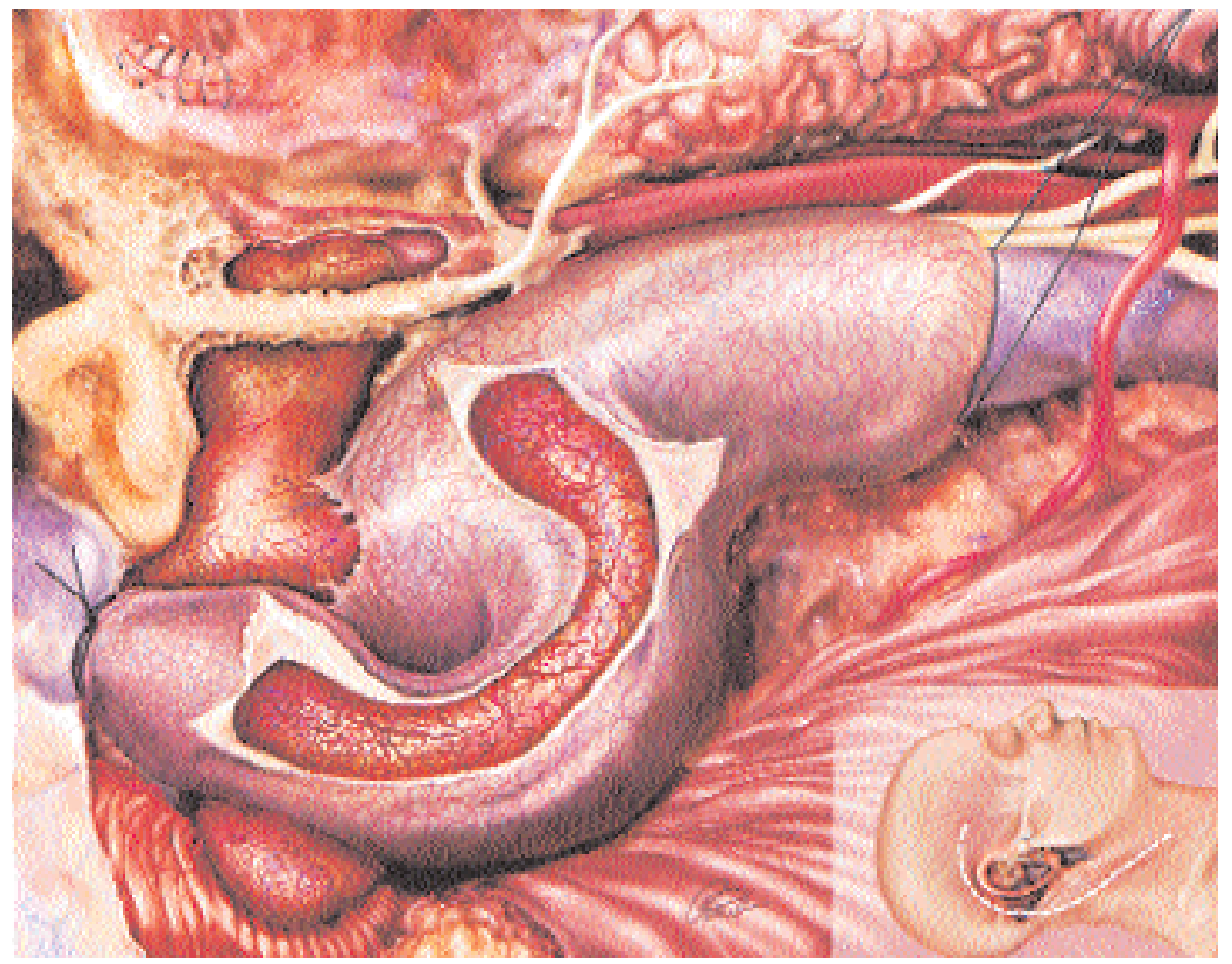

FIG. 2. Artist's illustration of total tumor isolation prior to resection. The tumor is exposed and dissected from the CA, lower cranial nerves, and facial nerve. The sigmoid sinus and the JV are then ligated and the sinus and jugular bulb are opened. The external ear canal is closed and the middle ear ossicle is removed. Inset: The postauricular incision used for this technique.

glomus jugulare requires special techniques in the handling of both arterial and venous dissection. Although paragangliomas engulf, adhere to, and receive blood from the ICA, with the aid of the operating microscope, we have been able to identify a plane of dissection to separate the tumor from the CA; thus, we found it unnecessary to sacrifice the CA or to reconstruct it. Only one patient had severe delayed vasospasm with thrombosis of the CA that required endovascular injection of urokinase.

Because exposure of tumors of the glomus jugulare requires neck dissection, associated tumors of the carotid body can be removed at the same time without additional morbidity or undue lengthening of the operating time.

Glomus jugulare tumors often shunt blood with high venous outflow. Accordingly, they should be handled as arteriovenous malformations. Therefore, venous drainage from the tumor should be preserved and the proximal end of the JV unligated until the tumor is isolated and its arterial supply is devascularized. We had to reestablish venous flow by using a shunt tube after premature ligation and sectioning of the $\mathrm{JV}$, which caused profuse bleeding from the tumor.

\section{Outcome of Surgically Treated Patients}

There was no instance of operative mortality in our series of patients, and gross-total removal was achieved in 24 of the 28 surgically treated patients. Immediate postoperative complications included one case of pneumothorax, one case of pulmonary embolism, and four cases of cerebrospinal fluid leak, which were treated through spinal drainage. In five patients infection developed at the closed external ear canal. Two of these infections progressed to osteomyelitis of the temporal bone; in one of these cases surgical debridement was required and the patient experienced meningitis. All infections were treated successfully. One patient had a retroperitoneal hematoma of undetermined cause that developed postoperatively. Another patient experienced hemiplegia postoperatively due to CA thrombosis, but recovered after endovascular injection of urokinase. One patient expe-

TABLE 5

Cranial nerve deficits in 28 patients who underwent surgery

\begin{tabular}{lcccc}
\hline \hline & \multicolumn{4}{c}{ No. of Cases } \\
\cline { 2 - 5 } \multicolumn{1}{c}{$\begin{array}{c}\text { Cranial Nerve } \\
\text { Deficit }\end{array}$} & Preop & Postop & $\begin{array}{c}\text { Follow } \\
\text { Up }\end{array}$ & $\begin{array}{c}\text { New } \\
\text { Permanent } \\
\text { Deficit }\end{array}$ \\
\hline II & 1 & 1 & 1 & 0 \\
III & 1 & 2 & 1 & 0 \\
IV & 0 & 2 & 1 & 1 \\
V & 6 & 8 & 7 & 1 \\
VI & 3 & 4 & 4 & 1 \\
VII & 11 & 16 & 13 & 2 \\
VIII & 13 & 14 & 14 & 1 \\
IX & 18 & 21 & 20 & 2 \\
X & 23 & 25 & 24 & 1 \\
XI & 12 & 16 & 13 & 1 \\
XII & 15 & 17 & 16 & 1 \\
Horner syndrome & 4 & 6 & 4 & 0 \\
\hline
\end{tabular}




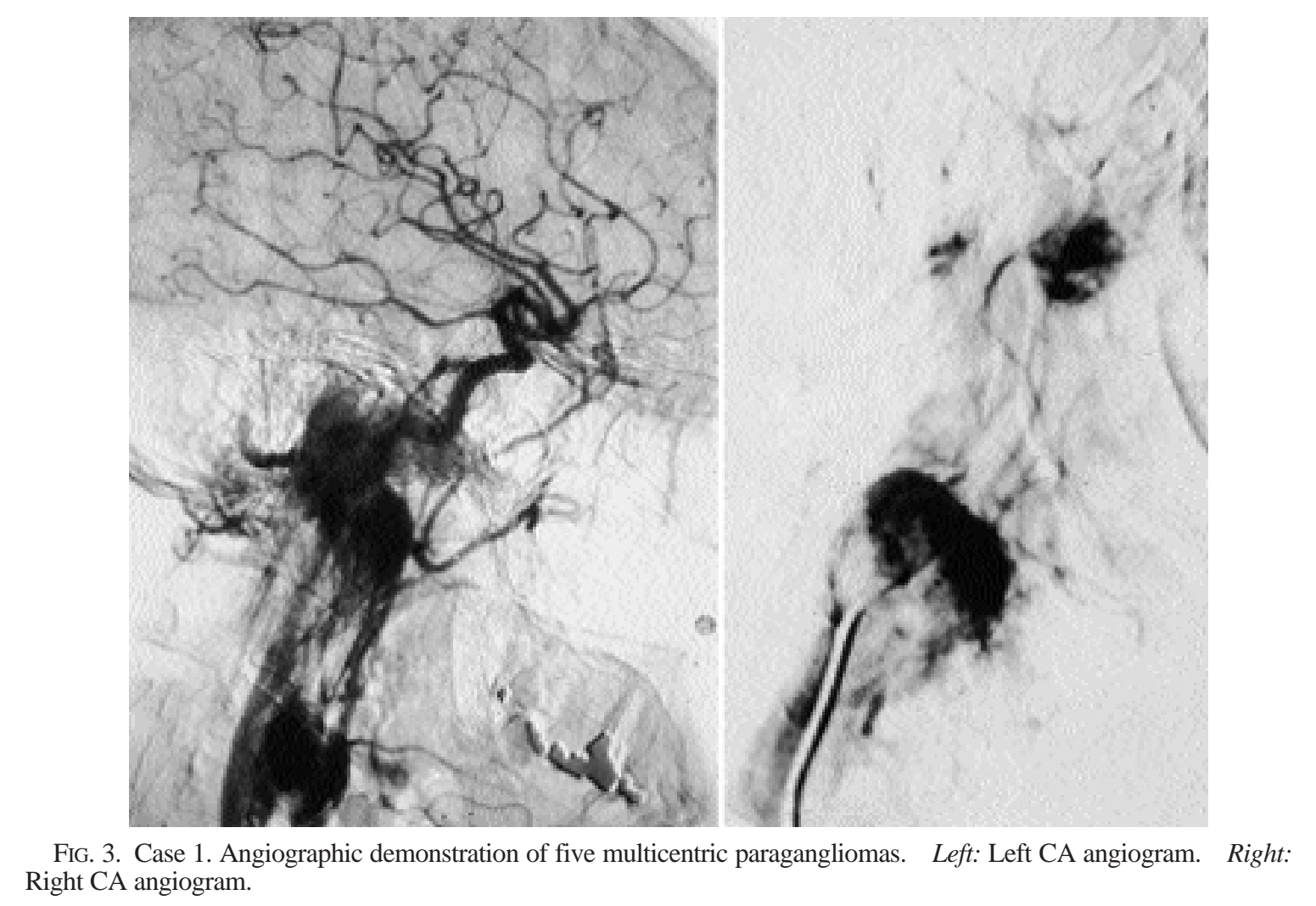

rienced transient hemiparesis. The course of cranial nerve function and new cranial nerve deficits are listed in Table 5. Five patients required a tracheostomy and four needed a gastrostomy. Two tumors recurred, one tumor that was malignant (see Case 2) and one benign tumor that recurred after 5 years. The average follow-up period of 38 months in this series is, unfortunately, relatively short. Many patients were lost to long-term follow up because they underwent surgery in various hospitals and we were not able to obtain continuous evaluations.

\section{Illustrative Cases}

\section{Case 1}

This 36-year-old woman presented with symptoms of hoarseness, neck pain, headache, and hypertension. On examination, she had decreased sensation in the first and second divisions of the fifth cranial nerve and palsies of the ninth and 10th nerves on the right side. Radiological studies demonstrated the presence of five paragangliomas: a glomus jugulare tumor on the right side, a glomus tympanicum tumor on the left side, bilateral carotid body tumors, and a sphenopalatine fossa tumor on the left side (Fig. 3).

Findings of an endocrinological workup in which a clonidine suppression test had been performed were positive for catecholamine secretion. The patient underwent resection of the tumors of the right glomus jugulare and the carotid body in the same setting. A tracheotomy tube, which had been placed preoperatively, was removed within 1 week, and the patient experienced transient facial nerve weakness on the right side from which she fully recovered. The facial nerve was deliberately not mobilized to minimize the risk of postoperative deficits. The ear canal was not closed to prevent the chance of hearing loss.

Two-and-a-half years later this patient underwent angiographic studies, which demonstrated marked enlargement of the carotid body tumor on the left side. She underwent resection of this tumor during which every attempt was made to preserve the lower cranial nerves and the facial nerve. Postoperatively, there was only a minor increase in secretions, and she was able to be discharged with a special diet. The problem intensified later, however, and required insertion of a tracheostomy tube and creation of a jejunostomy as well as eventual laryngeal reconstruction.

This case demonstrates the difficulty in treating multicentric paragangliomas. Patients with these tumors are at risk for bilateral morbid cranial nerve deficits (those of the seventh, eighth, or lower cranial nerves). Any of these occurrences decreases the patient's quality of life; therefore, appropriate management should be considered in advance and surgery or treatment should not be undertaken unless the tumor becomes fairly symptomatic and causes deficits or demonstrates clear growth. For surgical treatment, the surgeon should modify the technique to minimize the risk of bilateral deficits. These modifications include avoiding mobilization of the facial nerve, abandoning blind-sac closure of the external ear canal (associated with the infratemporal approach), performing intrabulbar dissection to prevent injury to the lower cranial nerves, accepting partial removal in an attempt to preserve lower cranial nerve function, and considering radiosurgery for the tumor on the opposite side, if treatment becomes necessary.

\section{Case 2}

This 55-year-old man underwent radiation therapy in 


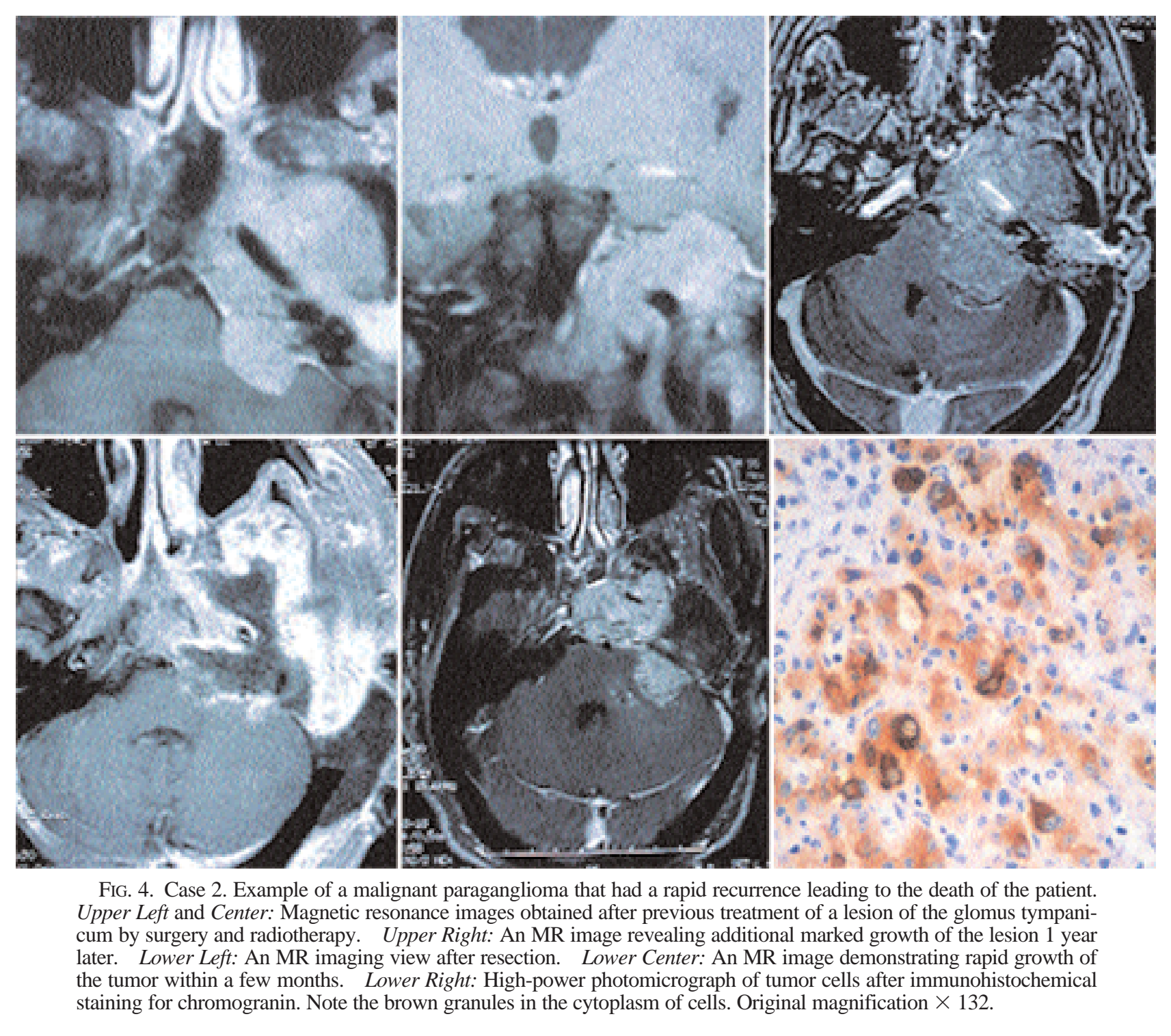

1976 after a biopsy confirmed a glomus tympanicum tumor in his left middle ear. He received 50 Gy in 15 fractions in a single field from the left side. In 1987, a right nephrectomy was performed for renal cell carcinoma. In 1997, the patient underwent embolization and a biopsy for a recurrent paraganglioma that caused lower cranial nerve deficits and facial nerve palsy. The facial nerve palsy progressed and diplopia developed. Because the tumor was enormous and had previously been treated with radiotherapy, it was considered inoperable and a trial of hydroxyurea was carried out with no response. When the patient presented to our institution, he had deficits in the fifth through 11th cranial nerves and paralysis of the 12th nerve with positive cerebellar signs. At the time the patient was receiving anticoagulation therapy for deep vein thrombosis. As part of the workup, he underwent a carotid balloon occlusion test to evaluate the collateral blood supply. The test caused ischemic deficits that took 2 months to resolve. The patient then underwent a total petrosectomy followed by total resection of the tumor. Postoperatively, he did well with no change in the earlier neurological findings. Immunohistochemical analysis confirmed a paraganglioma that stained positively for chromogranin with granules in the cytoplasm of cells, and chromosomal analysis demonstrated a nonclonal translocation that appeared randomly. Four months later, a large, rapidly growing recurrence of tumor became apparent, causing the patient's death (Fig. 4).

This case demonstrates the extensive neurological deficits that giant paragangliomas inflict. The surgical challenge lies in treating a giant tumor that involves the whole skull base and totally encases the CA. On the other hand, there is the ability to remove the tumor. The aggressive nature of the tumor recurrence defied any surgical excision and the case took an extremely malignant course. There is the question of whether administering radiation was effective for treating the small glomus tympanicum tumor in the ear 15 years earlier. This treatment may have been a factor in the aggressive transformation of this tumor into a malignant lesion. Radionecrosis of the temporal bone was also present on pathological findings in 1997. 


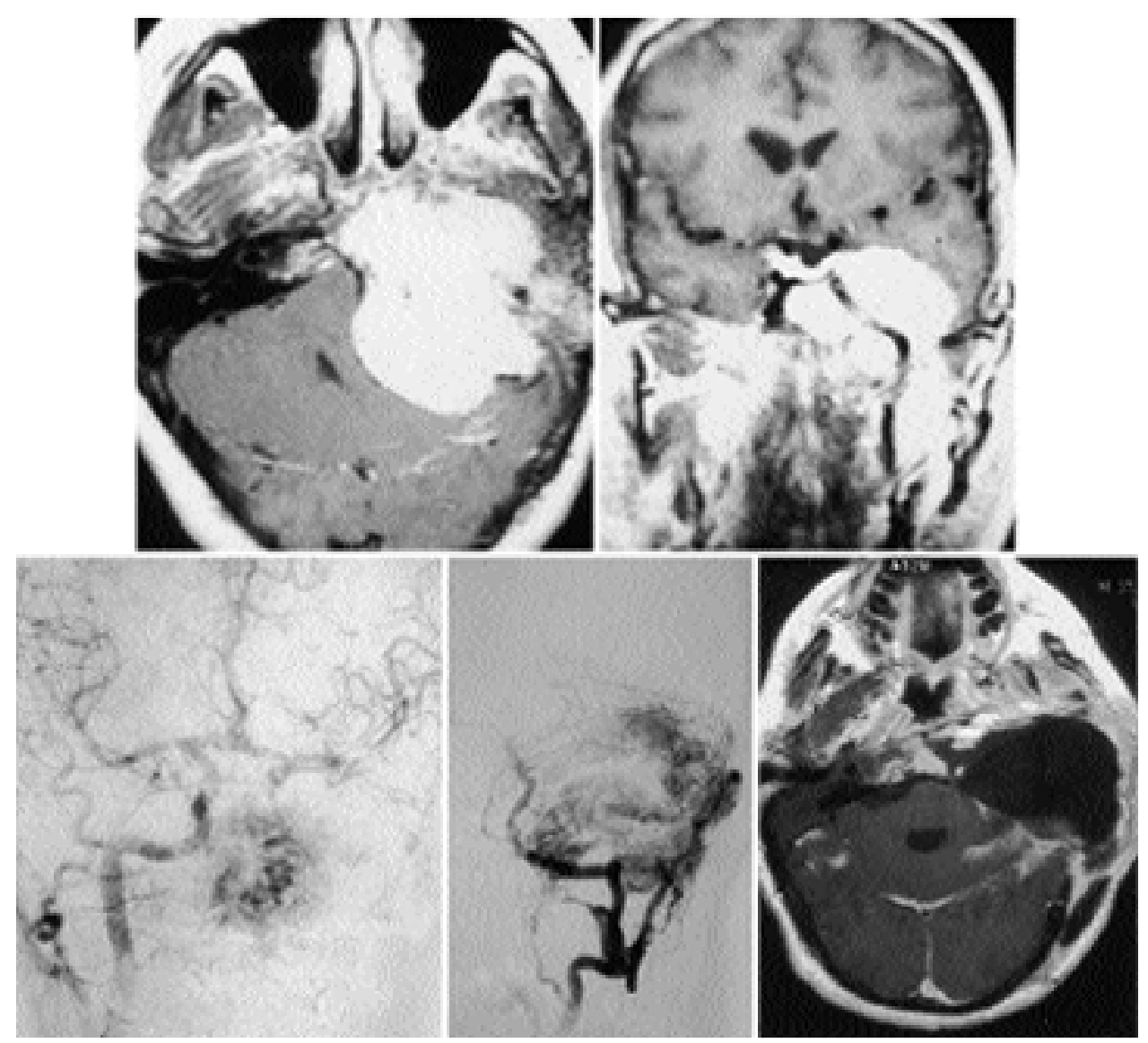

FIG. 5. Case 3. Example of a complex glomus jugulare tumor with catecholamine hypersecretion and giant size in a patient who previously received occlusion of the CA and experienced a morbid deficit. Upper Left and Right: Preoperative MR images. Lower Left: Contralateral CA angiogram demonstrating recruitment of blood supply from the patent CA. Lower Center: Vertebral artery angiogram revealing new blood supply from the vertebral and basilar arteries. Lower Right: Magnetic resonance image obtained after total resection of the tumor.

\section{Case 3}

In 1983, a 41-year-old man underwent partial resection of a tumor of the glomus jugulare. In 1991, he underwent a second surgery for a large recurrence of the tumor. The second operation was complicated by an intraoperative crisis in blood pressure, prolonged hospitalization with multiple cranial nerve deficits, pneumonia, aspiration, a cerebrospinal fluid leak, local tissue ischemia, and deep vein thrombosis. The patient was referred to us because his condition continued to deteriorate, he demonstrated metabolic wasting, and there was difficulty in managing his hypertension. When he presented to us in 1992, he had cranial nerve paralysis from the seventh to the 12th nerve on the left side. Imaging studies demonstrated a gigantic tumor that compressed the brainstem and posterior fossa, extended into the petrous canal and the cavernous sinus, and developed new vascularization from the opposite $\mathrm{CA}$ and vertebral artery after previous occlusion of the CA and embolization (Fig. 5). The patient underwent total removal of his tumor after preparation with a combined alpha and beta blocker. The tumor resection was followed by reconstruction with a free muscle flap. Pathological investigation confirmed the presence of a catecholamine-secreting tumor. The patient had a transient ocular motor dysfunction from which he re- covered, but the cranial nerve deficits that appeared earlier remained unchanged. He has had no recurrence as of the 7-year follow-up review.

This case shows the combination of factors that constitute a complex tumor of the glomus jugulare and presents the challenges of treating giant tumors, particularly those with catecholamine hypersecretion, brainstem compression, morbid deficits, and prior surgical, neurological, and systemic complications. This case emphasizes that current multidisciplinary advances have made the surgical treatment of such formidable lesions feasible and successful.

\section{Discussion}

\section{Giant Tumors}

The advent of computerized imaging, selective embolization, new surgical approaches, refinement of surgical techniques, and increased expertise have made it possible to treat glomus tympanicum and glomus jugulare tumors of moderate size surgically with good results and long-term disease control. $23,33,40,49$

In 1969, McCabe and Fletcher ${ }^{42}$ advocated the concept that the size and extent of the tumor are decisive for selecting the most appropriate form of therapy. Hence, authors 
of subsequent studies classified these tumors, emphasizing their size and intracranial extensions,,$^{20,28}$ and the operability of each tumor was decided by these factors. Still, it was wishful thinking that very large tumors could be removed.

Giant tumors are encountered rarely and are usually considered inoperable ${ }^{20,58}$ or are associated with high rates of morbidity and mortality. ${ }^{13,31,47,58}$ The two established classifications of these tumors are based mainly on tumor size with special emphasis on intracranial extension as a decisive factor for resectability. ${ }^{20,27}$ For example, Type D2 in the classification of Fisch and colleague ${ }^{20}{ }^{2}$ constitutes a tumor with an intracranial extension larger than $2 \mathrm{~cm}$ in diameter that mandates a two-stage surgery, and type D3 tumors are considered inoperable.

Similarly, in the classification of Glasscock and Jackson, ${ }^{28}$ a glomus jugulare tumor of Type IV extends beyond the petrous apex into the clivus or infratemporal fossa and may have an intracranial extension. Patel, et al., ${ }^{47}$ have voiced reservations about size alone being the critical factor and proposed that brainstem compression and vascular encasement should be considered because they can indicate higher complications and are, therefore, of prognostic value. The classification of Alford and Guilford ${ }^{1}$ defines a Stage IV tumor as one that has spread beyond the temporal bone, as indicated by the presence of multiple cranial nerve involvement. ${ }^{11}$ All these classifications distinguish a group of complex tumors that present challenges to treatment and are associated with adverse outcomes. Brown ${ }^{11}$ treated 42 Stage IV tumors, but never used surgery alone. Nineteen of the patients did not even undergo surgery, and another 19 underwent both surgery and radiotherapy.

The natural history of this tumor is generally believed to be benign and it may take years for adverse effects to appear. Unfortunately, the course of large tumors that cause neurological deficits is different. Thirty-five percent of patients in the series reported by Brown ${ }^{11}$ died within the 10year follow-up period.

Twenty-eight of our surgically treated patients harbored lesions that fulfilled the criteria for a giant tumor, and most of these tumors had a large intracranial, intradural extension that compressed the brainstem and extended into the petrous bone, the clivus, and the foramen magnum. Although surgery was lengthy, gross-total resection was feasible in the majority of patients without death or significant morbidity. We believe the key to resection is the total isolation of the tumor through a combined approach that exposes both the extracranial and intracranial extensions of the lesion. 2,23,47 Thus, we hold in disfavor the staging of surgery to tackle the extradural and intradural portions of the tumor separately.

\section{Multiple Paragangliomas}

Multiple paragangliomas present the greatest challenge to treating complex paragangliomas because the treatment decision is not made based on a single tumor, but on the quality and length of the patient's life. Whether to treat, when to treat, which tumors to treat, with which modality (surgery or radiation) of treatment, and in which sequence - all are questions that must be addressed at the first evaluation and thoroughly considered throughout the patient's follow up. The surgeon must try to prevent the consequences of multiple bilateral cranial nerve deficits, that is, severe morbidity and a poor quality of life.
Multicentricity associated with glomus tumors is reported to be more than $10 \% .23,31,58,65$ Familial cases, most of which have involved fathers and daughters, have a much higher incidence of multicentricity, up to 55\%.62,64 The rate of multicentricity in our patients was $34.5 \%$, but none of our patients had the familial form of the disease. The possibility of multicentricity, which is significant in number and extremely high in cases of the familial form, mandates that each patient who is given a diagnosis of paraganglioma in one location must be fully evaluated for other lesions. Most multicentric tumors, however, are bilateral tumors of the carotid body. ${ }^{44,48}$

The second lesion associated with a tumor of the glomus jugulare is usually a tumor of the ipsilateral carotid body. Because this tumor is also exposed during the approach to the glomus jugulare tumor, and the lower cranial nerves on that side are dissected at the lower pole of the glomus jugulare tumor, we believe that a unilateral carotid body tumor is best excised during the same operation.

A few cases of bilateral glomus jugulare tumors associated with carotid body tumors have been reported. ${ }^{62-65}$ These cases illustrate the paramount need for accurate evaluation, including family evaluation and counseling. Because of the high morbidity associated with deficits of the bilateral seventh nerve, the eighth nerve, and the lower cranial nerves, and the poor quality of life projected for any patient having these deficits, either from the tumor or as a consequence of treatment, the bilateral form of these tumors requires the most thoughtful and thorough evaluation and counseling with clear patient expectations. We agree with the recommendations of Van Der Mey and colleagues ${ }^{64}$ that the opposite side should be treated surgically only if the first resection did not cause essential cranial nerve palsy. Otherwise, these authors refrain from surgery and favor close follow-up review. Unfortunately, our patient who experienced a rapid growth that prompted surgery on the opposite side for a carotid body tumor had prolonged dysfunction of swallowing and pulmonary complications.

For patients with multiple paragangliomas, the surgical technique should also be modified. The classic infratemporal approach of Fisch and colleagues, ${ }^{19,20}$ which includes facial nerve transposition, blind-sac closure of the ear canal, and subsequent loss of air conduction hearing, should be modified to minimize the risk of facial nerve palsy and, at least, conductive hearing loss. ${ }^{2}$ Intrabulbar dissection is particularly critical in these cases to maximize the chance of preserving lower cranial nerves on the operated side so that one could, in the future, treat the opposite side. The surgeon must also consider the role of radiosurgery for bilateral tumors. Radiation therapy has long been used to treat glomus tumors, particularly those that are partially removed or have recurred. ${ }^{14,15,52}$ Glomus tumors are known to be radioresistant, however, and the effect of radiation is often the induction of fibrosis, mainly along the vessels supplying the tumors. ${ }^{12,24}$ In addition, persistent viable tumors are often present long after radiotherapy. Radiation therapy has also been associated with long-term side effects that include temporal bone osteonecrosis, development of a new malignancy, and brain injury. ${ }^{14,37}$

Early reports of stereotactic radiosurgery for glomus tumors are encouraging. ${ }^{17,21,36,39}$ It appears to be very effective if the target size is within the optimal size for radiosurgery. All our patients harbored tumors that were too large for this 
treatment. The preliminary results of radiosurgery suggest a symptomatic improvement of cranial nerve function. ${ }^{39}$ If radiosurgery is proven to be effective with few complications from cranial nerve deficits, it will be a great complement to the treatment of bilateral glomus jugulare tumors and residual tumors left after resection of giant tumors. The concern over the high level of morbidity associated with multiple cranial nerve deficits and the capability of radiosurgery to treat unresectable residual tumor have led to the recommendation of this treatment modality for petroclival meningiomas. ${ }^{15}$

\section{Cranial Nerve Deficits}

Surgery of tumors of the glomus jugulare has been haunted by the complication of postoperative deficits of the lower and other cranial nerves. . $^{13,29,30,64,65}$ Although this finding has been exploited by the proponents of radiation therapy, ${ }^{10,16,60}$ in patients with giant tumors the lower cranial nerves are already seriously affected. Patients may require additional pulmonary cautions during the early postoperative weeks to avoid aspiration. We believe, however, that intrabulbar dissection can help preserve the function of the lower cranial nerves. Unfortunately, this technique is not useful for tumors that penetrate the outer layer of the venous wall or that actually invade the cranial nerves. ${ }^{40,55}$

\section{Vascular Considerations}

The literature contains many statements with regard to tumor invasion of the arterial wall and sacrifice of the CA either in advance or intraoperatively. Reports also document particular difficulties encountered in treating the petrous carotid segment. ${ }^{4,31}$ Sacrifice and reconstruction of the CA, however, have both been associated with major risks. ${ }^{20,64}$ Two patients in the series of Jackson and colleagues ${ }^{31}$ suffered intraoperative trauma to the ICA, which was associated with massive stroke and subsequent death. We believe, however, that a controlled ICA dissection can be performed. A plane can be developed between the adventitia and the tumor and, with the aid of the microscope, the feeding vessel from the CA can be coagulated and sectioned. We do not believe that deliberate sacrifice of the CA is warranted. The risk of permanent CA occlusion is high, even in patients who pass a balloon occlusion test. ${ }^{28,45}$ Our experience in this series reinforces our previous commitment to preserving the CA. ${ }^{3,9,45}$ The balloon occlusion test itself carries a complication rate of 3.7\%. ${ }^{61}$ Similarly, revascularization with a bypass carries the risk of ischemic complications. ${ }^{54,56,59}$ Previous occlusion or embolization of the CA creates a new blood supply from the vertebral artery and the internal petrous $\mathrm{CA}$, which increases the risk of arterial dissection.

There has been little discussion about managing venous outflow. In this respect, tumors of the glomus jugulare must be considered to be arteriovenous malformations. A considerable amount of blood is shunted through the collateral venous plexus, particularly if the sigmoid sinus is totally occluded. Ligation of the JV must be delayed until the end of the procedure to maintain good venous drainage from the tumor and to achieve full devascularization from the arterial side and the surrounding area before the final interruption of blood drainage.

\section{Hormone-Secreting Paragangliomas}

Paragangliomas are sometimes associated with the secretion of a wide variety of neuropeptide hormones, including adrenocorticotropic hormone, serotonin, catecholamine, and dopamine. Thus far the focus has been on the secretion

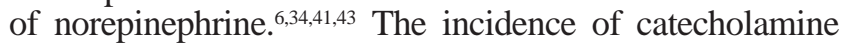
secretion is approximately $4 \% .^{11,32}$ Frequently, these tumors have a low level of secretion that is not recognized clinically ${ }^{43}$ Because of the potentially serious complications of an intra- or perioperative hypertension crisis, we agree that screening for excess catecholamine secretion is indicated in all patients with tumors of the glomus jugulare..$^{28,43,53} \mathrm{In}$ patients with catecholamine secretion and multicentric tumors, selective intravenous sampling is warranted to establish the site of catecholamine production. ${ }^{46}$ The actual treatment and the duration of prophylaxis depends on the level of catecholamine secretion and its source. Patients with these tumors might also harbor adrenal norepinephrine-secreting tumors. Thus, adrenal imaging is particularly important in patients with hypersecreting tumors. Patients with confirmed hypersecreting tumors (patients in whom the catecholamine level is four times higher than normal) require preparation with an alpha and beta catecholamine blocker such as labetalol before surgery, angiography, or embolization. Beta blockers should not be used before alpha blockers. An unopposed alpha agonist introduced into the setting of a beta blocker can cause severe hypertension and cardiac crisis. ${ }^{34}$ Recently, calcium-channel blockers and magnesium sulfate were recommended for patients with pheochromocytoma. $^{34,50}$

Among the other various secretions of paragangliomas, serotonin and its precursor, 5-hydroxytryptamine, are rarely associated with the clinical presentation of a carcinoid syndrome. ${ }^{18,34}$ The release of histamine and bradykinin during surgical manipulation can cause profound hypotension and even shock, with bronchial constriction that does not respond to conventional therapy. ${ }^{34}$

\section{Malignant Paragangliomas}

Malignant paragangliomas are seldom reported. Histological criteria do not predict the clinical behavior of paragangliomas, and their malignant potential is notoriously difficult to predict. Aside from overt metastasis, few if any microscopic features exist that allow the surgical pathologist to determine reliably whether a paraganglioma is benign or malignant. Hence, malignancy is determined more by a clinical course of rapid, aggressive growth, which is usually associated with anemia and the presence of metastasis. ${ }^{7,38}$ By 1980, there were 27 documented cases, ${ }^{66}$ and a few additional ones have been reported since the paper by Zak and Lawson. ${ }^{66}$ The natural history is usually one of rapid death, as can be seen in one of our patients. The most common sites of metastasis are in the neck, lymph nodes, and lung, but other visceral metastases have also been noted. ${ }^{66} \mathrm{~A}$ review of the current literature shows recommendations for the use of electron microscopy and immunohistochemical studies with a panel of neuroendocrinological markers. ${ }^{35}$ Clinically, malignant paragangliomas of a proven metastasis express considerably lower levels of neuropeptide. ${ }^{38}$ The karyotyping used in our patient constitutes the first reported instance in which malignancy was determined through this method, but it did not show the 
abnormalities seen in the familial form, which is associated with the presence of the disease haplotype at chromosome band $11 \mathrm{q} 23 .{ }^{26,48}$ Obviously, the prognosis for patients with malignant paragangliomas is extremely poor, and the effects of chemotherapy and radiation treatment have not been encouraging. ${ }^{7}$

\section{Conclusions}

Despite the challenges encountered in treating complex glomus jugulare tumors, resection is indicated and successful. Multiple tumors mandate a treatment plan that addresses the risk of bilateral cranial nerve deficits. The intrabulbar dissection technique can be used with any tumor as long as the tumor itself has not penetrated the wall of the jugular bulb or infiltrated the cranial nerves. Tumors that hypersecrete catecholamine require perioperative management and malignant tumors carry a poor prognosis.

\section{References}

1. Alford BR, Guilford FR: A comprehensive study of tumors of the glomus jugulare. Laryngoscope 72:765-787, 1962

2. Al-Mefty O, Fox JL, Rifai A, et al: A combined infratemporal and posterior fossa approach for the removal of giant glomus tumors and chondrosarcomas. Surg Neurol 28:423-431, 1987

3. Anand VK, Leonetti JP, Al-Mefty O: Neurovascular considerations in surgery of glomus tumors with intracranial extensions. Laryngoscope 103:722-728, 1993

4. Andrews JC, Valavanis A, Fisch U: Management of the internal carotid artery in surgery of the skull base. Laryngoscope 99: 1224-1229, 1989

5. Arnautovic KI, Al-Mefty O, Angtuaco E, et al: Dural arteriovenous malformations of the transverse/sigmoid sinus acquired from dominant sinus occlusion by a tumor: report of two cases. Neurosurgery 42:383-388, 1998

6. Azzarelli B, Felten S, Muller J, et al: Dopamine in paragangliomas of the glomus jugulare. Laryngoscope 98:573-78, 1988

7. Bojrab DI, Bhansali SA, Glasscock ME III: Metastatic glomus jugulare: long-term followup. Otolaryngol Head Neck Surg 104: 261-264, 1991

8. Borba LA, Al-Mefty O: Intravagal paragangliomas: report of four cases. Neurosurgery 38:569-575, 1996

9. Bowles AP, Al-Mefty O: The cavernous carotid artery: preservation is the best means of reconstruction. Clin Neurosurg 42: 154-170, 1995

10. Boyle JO, Shimm DS, Coulthard SW: Radiation therapy for paragangliomas of the temporal bone. Laryngoscope 100:896-901, 1990

11. Brown JS: Glomus jugulare tumors revisited: a ten-year statistical follow-up of 231 cases. Laryngoscope 95:284-288, 1985

12. Carrasco V, Rosenman J: Radiation therapy of glomus jugulare tumors. Laryngoscope 103 (Suppl 60):23-27, 1993

13. Cece JA, Lawson W, Biller HF, et al: Complications in the management of large glomus jugulare tumors. Laryngoscope 97: 152-157, 1987

14. Cole JM, Beiler D: Long-term results of treatment for glomus jugulare and glomus vagale tumors with radiotherapy. Laryngoscope 104:1461-1465, 1994

15. Couldwell WT, Fukushima T, Giannotta SL, et al: Petroclival meningiomas: surgical experience in 109 cases. J Neurosurg 84: 20-28, 1996

16. Cummings BJ, Beale FA, Garrett PG, et al: The treatment of glomus tumors in the temporal bone by megavoltage radiation. Cancer 53:2635-2640, 1984
17. Eustacchio S, Leber K, Trummer M, et al: Gamma knife radiosurgery for glomus jugulare tumours. Acta Neurochir 141:811-818, 1999

18. Farrior JB III, Hyams VJ, Benke RH, et al: Carcinoid apudoma arising in a glomus jugulare tumor: review of endocrine activity in glomus jugulare tumors. Laryngoscope 90:110-119, 1980

19. Fisch U: Infratemporal fossa approach to tumours of the temporal bone and base of the skull. J Laryngol Otol 92:949-967, 1978

20. Fisch U, Fagan P, Valavanis A: The infratemporal fossa approach for the lateral skull base. Otolaryngol Clin North Am 17: $513-552,1984$

21. Foote RL, Coffey RJ, Gorman DA, et al: Stereotatic radiosurgery for glomus jugulare tumors: a preliminary report. Int J Radiat Oncol Biol Phys 38:491-495, 1997

22. Forest JA III, Jackson CG, McGrew BM: Long-term control of surgically treated glomus tympanicum tumors. Otol Neurotol 22: 232-236, 2001

23. Green JD Jr, Brackmann DE, Nguyen CD, et al: Surgical management of previously untreated glomus jugulare tumors. Laryngoscope 104:917-921, 1994

24. Hawthorne MR, Makek MS, Harris JP, et al: The histopathological and clinical features of irradiated and nonirradiated temporal paragangliomas. Laryngoscope 98:325-331, 1988

25. Heilman CB, Robertson JH, Gardner G, et al: Surgical management of glomus jugulare tumors, in Schmidek HH (ed): Schmidek \& Sweet Operative Neurosurgical Techniques: Indications, Methods, and Results, ed 4. Philadelphia: WB Saunders, 2001, Vol 1, pp 1041-1055

26. Heutink P, van der Mey AG, Sandkuijl LA, et al: A gene subject to genomic imprinting and responsible for hereditary paragangliomas maps to chromosome 11q23-qter. Hum Mol Genet 1:7-10, 1992

27. Jackson CG: Basic surgical principles of neurotologic skull base surgery. Laryngoscope 103:29-44, 1993

28. Jackson CG: Neurotologic skull base surgery for glomus tumors. Diagnosis for treatment planning and treatment options. Laryngoscope 103 (Suppl 60): 17-22, 1993

29. Jackson CG, Cueva RA, Thedinger BA, et al: Conservation surgery for glomus jugulare tumors: the value of early diagnosis. Laryngoscope 100:1031-1036, 1990

30. Jackson CG, Cueva RA, Thedinger BA, et al: Cranial nerve preservation in lesions of the jugular fossa. Otolaryngol Head Neck Surg 105:687-693, 1991

31. Jackson CG, Glasscock ME III, McKennan KX, et al: The surgical treatment of skull-base tumors with intracranial extension. Otolaryngol Head Neck Surg 96:175-185, 1987

32. Jackson CG, Harris PF, Glasscock ME III, et al: Diagnosis and management of paragangliomas of the skull base. Am J Surg 159:389-393, 1990

33. Jackson CG, Haynes DS, Walker PA, et al: Hearing conservation in surgery for glomus jugulare tumors. Am J Otol 17:425-437, 1996

34. Jensen NF: Glomus tumors of the head and neck: anesthetic considerations. Anesth Analg 78:112-119, 1994

35. Johnson TI, Zarbo RJ, Lloyd RV, et al: Paragangliomas of the head and neck: immunohistochemical neuroendocrine and intermediate filament typing. Mod Pathol 1:216-223, 1988

36. Jordan JA, Roland PS, McManus C, et al: Stereotactic radiosurgery for glomus jugulare tumors. Laryngoscope 110:35-38, 2000

37. Lalwani AK, Jackler RK, Gutin PH: Lethal fibrosarcoma complicating radiation therapy for benign glomus jugulare tumor. Am J Otol 14:398-402, 1993

38. Linoilla RI, Lack EE, Steinberg SM, et al: Decreased expression of neuropeptides in malignant paragangliomas: an immunohistochemical study. Hum Pathol 19:41-50, 1988

39. Liscak R, Vladyka V, Simonova G, et al: Leksell gamma knife radiosurgery of the tumor glomus jugulare and tympanicum. Stereotact Funct Neurosurg 70 (Suppl 1):152-160, 1998 
40. Makek M, Franklin DJ, Zhao JC, et al: Neural infiltration of glomus temporale tumors. Am J Otol 11:1-5, 1990

41. Matishack MZ, Symon L, Cheeseman A, et al: Catecholamine-secreting paragangliomas of the base of the skull. Report of two cases. J Neurosurg 66:604-608, 1987

42. McCabe BF, Fletcher M: Selection of therapy of glomus jugulare tumors. Arch Otolaryngol 89:156-159, 1969

43. Nelson MD, Kendall BE: Intracranial catecholamine secreting paragangliomas. Neuroradiology 29:277-282, 1987

44. Ophir D: Familial multicentric paragangliomas in a child. J Laryngol Otol 105:376-380, 1991

45. Origitano TC, Al-Mefty O, Leonetti JP, et al: Vascular considerations and complications in cranial base surgery. Neurosurgery 35:351-363, 1994

46. Palubinskas AJ, Roizen MF, Conte FA: Localization of functioning pheochromocytomas by venous sampling and radioenzymatic analysis. Radiology 136:495-496, 1980

47. Patel SJ, Sekhar LN, Cass SP, et al: Combined approaches for resection of extensive glomus jugulare tumors: a review of 12 cases. J Neurosurg 80:1026-1038, 1994

48. Petropoulos AE, Luetje CM, Camarate PJ, et al: Genetic analysis in the diagnosis of familial paragangliomas. Laryngoscope 110: 1225-1229, 2000

49. Poe DS, Jackson G, Glasscock ME, et al: Long-term results after lateral cranial base surgery. Laryngoscope 101:372-378, 1991

50. Proye C, Thevenin D, Cecat P, et al: Exclusive use of calcium channel blockers in preoperative and intraoperative control of pheochromocytomas: hemodynamics and free catecholamine assays in ten consecutive patients. Surgery 106:1149-1154, 1989

51. Rosenwasser H: Carotid body tumor of the middle ear and mastoid. Arch Otolaryngol 41:64-67, 1945

52. Schild SE, Foote RL, Buskirk SJ, et al: Results of radiotherapy for chemodectomas. Mayo Clin Proc 67:537-540, 1992

53. Schwaber MK, Glasscock ME, Nissen AJ, et al: Diagnosis and management of catecholamine secreting glomus tumors. Laryngoscope 94:1008-1015, 1984

54. Sekhar LN, Sen CN, Jho HD: Saphenous vein graft bypass of the cavernous internal carotid artery. J Neurosurg 72:35-41, 1990

55. Sen C, Hague K, Kacchara R, et al: Jugular foramen: microscopic anatomic features and implications for neural preservation with reference to glomus tumors involving the temporal bone. Neurosurgery 48:838-848, 2001
56. Sen C, Sekhar LN: Direct vein graft reconstruction of the cavernous, petrous, and upper cervical internal carotid artery: lessons learned from 30 cases. Neurosurgery 30:732-743, 1992

57. Simko TG, Griffin TW, Gerdes AJ, et al: The role of radiation therapy in the treatment of glomus jugulare tumors. Cancer 42: 104-106, 1978

58. Spector GJ, Sobol S: Surgery for glomus tumors at the skull base. Otolaryngol Head Neck Surg 88:524-530, 1980

59. Spetzler RF, Fukushima T, Martin N, et al: Petrous carotid-tointradural carotid saphenous vein graft for intracavernous giant aneurysm, tumor, and occlusive cerebrovascular disease. J Neurosurg 73:496-501, 1990

60. Springate SC, Weichselbaum RR: Radiation or surgery for chemodectoma of the temporal bone: a review of local control and complications. Head Neck 12:303-307, 1990

61. Tarr RW, Jungreis CA, Horton JA, et al: Complications of preoperative balloon test occlusion of the internal carotid arteries: experience in 300 cases. Skull Base Surg 1:240-244, 1991

62. Van Baars F, van den Broek P, Cremers C, et al: Familial nonchromaffinic paragangliomas (glomus tumors): clinical aspects. Laryngoscope 91:988-996, 1981

63. Van Der Borden J: Bilateral non-chromaffin tympano-jugular paraganglioma. J Laryngol Otol 81:445-448, 1967

64. Van Der Mey AG, Frijns JH, Cornelisse CJ, et al: Does intervention improve the natural course of glomus tumors? A series of 108 patients seen in a 32-year period. Ann Otol Rhinol Laryngol 101:635-642, 1992

65. Woods CI, Strasnick B, Jackson CG: Surgery for glomus tumors: the Otology Group Experience. Laryngoscope 103 (Suppl 60): 65-70, 1993

66. Zak FG, Lawson W: The Paraganglionic Chemoreceptor System: Physiology, Pathology, and Clinical Medicine. New York: Springer-Verlag, 1982, pp 339-391

Manuscript received March 12, 2002.

Accepted in final form August 7, 2002.

Address reprint requests to: Ossama Al-Mefty, M.D., Department of Neurosurgery, University of Arkansas for Medical Sciences, 4301 West Markham, Slot 507, Little Rock, Arkansas 72205. email: keelandamye@uams.edu. 